\title{
Los Núcleos de Intervención Participativa (NP): análisis de tres experiencias
}

Joan Font *

Los Núcleos de Intervención Participativa (NIP) son una de las múltiples propuestas que han aparecido en medio del debate en torno a posibles nuevos mecanismos de participación ciudadana. Los NIP tratan de integrar y de recoger lo mejor de mecanismos tan dispares como asambleas, jurados, consejos consultivos y encuestas de opinión, para ofrecer otra posibilidad de que las voces de los ciudadanos se escuchen en el proceso de toma de decisiones públicas. Los NIPs son grupos de personas elegidas al azar que, tras un proceso de información plural y de deliberación colectiva, emiten un dictamen sobre un problema colectivo que exige una respuesta pública.

Este artículo pretende, en primer lugar, explicar qué son y cómo funcionan los NIP. A continuación, explicaremos tres experiencias de aplicación de este procedimiento en Guipúzcoa $y$, a partir de las mismas, realizaremos un breve análisis de las potencialidades, los condicionantes y los límites de la posible aplicación de los NIPs como instrumento de participación ciudadana.

Aunque la justificación de la necesidad de encontrar nuevos mecanismos de participación democrática ha quedado ya realizada en otro artículo de este mismo número ', es preciso añadir un par de observaciones introductorias. Se ha dicho que participación política eran todos aquellos actos dirigidos a seleccionar a nuestros representantes o a influir en la toma de decisiones públicas. El primer grupo de actividades encuentra su institucionalización en los procesos electorales con todo lo que ello implica: un período de discusión y debate público, unas reglas para la toma de decisiones claras y un proceso de movilización impulsado por el Estado, los partidos y otras organizaciones, que lleva a que la mayor parte de ciudadanos participe. Por el contrario, la participación dirigida a influir en la toma de decisiones forma parte de un universo mucho menos regulado y donde por tanto priman en mayor medida los acuerdos informales, las presiones indirectas o las alianzas cambiantes ${ }^{2}$. No hay período formalizado de debate público que llegue a la mayoría de los ciudadanos, no está escrito a quien hay que escuchar y a quien no, la movilización alcanza sólo, general- mente, a los que pertenecen a organizaciones más o menos afectadas. Es decir que, a pesar del papel crucial que juega la intervención de las asociaciones, el debate se reduce a la minoría organizada y la participación episódica de los demás tiene lugar a través de mecanismos informales, donde la información exhaustiva y el debate sereno son la excepción y no la regla. Sin pretender que todo el mundo intervenga en cada decisión, la búsqueda de mecanismos que, como los NIP, permitan la participación de sectores más plurales, que ésta se realice en condiciones de información y debate y con mecanismos claros de funcionamiento, resulta un camino en una dirección interesante.

A pesar de la percepción generalizada de la necesidad de mejorar los mecanismos de participación, esto no implica que vaya a haber acuerdo sobre su intensidad, sus mecanismos ideales de aplicación o su combinación con la lógica de la representación. El conflicto en torno a las formas de organización política, que en otras épocas tomara forma principal en términos de debate entre democracia y dictadura, entre sufragio censitario y universal o entre monarquía y república, puede girar en el futuro alrededor del cuánto y el cómo de la participación ciudadana en la toma de decisiones. No estamos pues sólo ante un debate sobre mecanismos de mejora de la gestión pública, sino ante una opción con profundas implicaciones políticas, donde los objetivos a conseguir definirán ls prioridades que se establezcan.

\section{I. ¿Qué son los NIP?}

Los NIP son una técnica para la intervención de los ciudadanos en los procesos de toma de decisiones públicas. Su origen intelectual se encuentra en un grupo de sociólogos alemanes que, tras algunas experiencias exitosas en su país, ha empezado a exportar el producto a otros contextos ${ }^{3}$. Además de aplicaciones en Gran Bretaña, Japón o Israel, los NIP han sido experimentados en Guipúzcoa y son objeto de escrutinio por parte de otras administraciones españolas ${ }^{4}$. 
Los NIP son grupos, organizados y moderados por técnicos externos, de unos 25 ciudadanos escogidos aleatoriamente, que tras un periodo de información y debate a tiempo completo sobre un tema que exige una respuesta pública, emiten un dictamen que tendrá un carácter formalmente no vinculante para la institución pública que lo ha encargado. Diseccionemos por partes esta definición.

Los NIP se estructuran a partir de grupos de ciudadanos que cuentan con alrededor de 25 miembros. Para tomar una misma decisión pueden constituirse uno, dos o muchos NIPs según el tamaño y la heterogeneidad de la población afectada, aunque el dictamen final será el resultado de las opiniones de todos ellos. El tamaño ideal del NIP, aunque debe tomarse más como indicación que como dogma, parte de la voluntad de encontrar un equilibrio entre dos factores: un número mínimo de gente para asegurar la discusión y para que el proceso de información no sea excesivamente costoso, y una cifra que permita un verdadero diálogo donde todo el mundo participe. De hecho, para asegurar que esto ocurra, la discusión tiene lugar en muchas ocasiones en subgrupos de 5 personas.

La organización y la moderación de los NIP corre a cargo de una entidad externa, que garantice cierta independencia respecto a la institución que lo encarga y un tratamiento profesional de la selección de la muestra, las dinámicas de grupos y de la elaboración del cuestionario y el informe final.

Ia selección de los ciudadanos se realiza a través de un muestreo aleatorio entre la población afectada. Este es un proceso sencillo si se trata de una única localidad y mucho más complejo si involucra a un territorio mayor. Además de los ciudadanos elegidos como miembros "titulares" del NIP, se escoge una segunda y tercera muestra de "suplentes" para prever los rechazos a participar, que por imposibilidad o desinterés existen siempre.

La participación de los ciudadanos en el NIP se desarrolla generalmente a lo largo de entre 3 y 5 dias a tiempo completo. Esto permitirá la información detallada y el debate, pero implica, obviamente, que se trate de una actividad remunerada, en la que se cubran gastos y ganancias no percibidas. Además, se da a los participantes todo el apoyo posible para obtener permisos de sus empresas, cuidar a los niños, etc...

La puesta en marcha del NIP es comunicada al conjunto de la población vía publicidad y medios de comunicación. Entonces se pone en marcha la recogida de propuestas de todos aquellos que se sientan afectados y tengan algo que decir, para que manifiesten su voluntad de informar a los miembros de los NIP. Generalmente, se tratará sobre todo de organizaciones ciudadanas, que quieran manifestar sus opiniones al respecto. Además de a estos grupos, los NIP escucharán también a expertos en cada uno de los temas afectados. Tras cada presentación habrá preguntas y luego debate entre los miembros del NIP, divididos en subgrupos de 5.

Al final de este período, la organización pasará un cuestionario a cada uno de los miembros, que trate de reflejar todos los temas discutidos a lo largo de las sesiones y que permita elaborar unas recomendaciones finales. A partir de las respuestas, se elaborará un informe descriptivo de los resultados, que contará con la asesoría y la aprobación de una comisión de los participantes. En tanto que la capacidad legal de decidir pertenece a los representantes políticos, el informe recoge las preferencias de los ciudadanos, pero no tiene carácter vinculante.

\section{Tres NIPs en Guipúzcoa}

Las primeras experiencias de NIPs en nuestro entorno han tenido lugar en Guipúzcoa. La traumática experiencia que supuso la construcción de la autovía de Leizarán, con los costes económicos y políticos que supuso, una cultura con una gran tradición participativa y la propia complejidad de la vida política vasca y guipuzcoana, favorecieron probablemente que fuera alli donde se desarrollaran los primeros NIPs. El interés en el tema del Arateko (Defensor del Pueblo) vasco, que actuó como impulsor y difusor de la idea durante el año 1991, fue también fundamental. Entre 1992 y 1994 tendrán lugar estos tres procesos, que describiremos a continuación y cuyo análisis más detenido realizaremos en el próximo apartado.

El primero de estos NIPs se realiza en Idiazabal, una pequeña comunidad de la comarca del Goierri. Es la experiencia más reducida y la que tiene lugar en un entorno de mayor consenso, desde el momento de su planificación y a lo largo de todo su desarrollo. El equipo de fútbol local juega en un terreno cedido por una empresa y que por tanto ésta puede reclamar en cualquier momento. En la población existía una percepción generalizada de que ésta era una actividad social y deportiva fundamental y que era preciso encontrar un emplazamiento más idoneo, para que desapareciera la espada de Damocles que suponía la posible reclamación del terreno por la empresa en cualquier momento. Era preciso encontrar un nuevo emplazamiento $y$, aunque en el municipio había varios que contaban con los requisitos necesarios, todos ellos pertenecían a propietarios que no estaban interesados en venderlos. A falta de claros argumentos técnicos o políticos a favor de uno u otro emplazamiento (ahabíamos expuesto las alternativas y teníamos un follón de miedo", declara el Alcalde), el ayuntamiento opta por utilizar un NIP para tomar la decisión. Al interés por solucionar el problema, se une en este caso una preocupación más de fondo por la escasa implicación ciudadana en los temas municipales, ante lo cual el NIP aparece también como revulsivo para generar un mayor debate público. 
Entre los elementos más interesantes de este caso aparece la primera idea que dará el NIP: todas las alternativas sugeridas por el ayuntamiento se encontraban en la zona sur del pueblo, afectada recientemente por una autopista, por lo que el NIP sugiere buscar emplazamientos en la zona norte para repartir cargas. Efectivamente, la propuesta final se encontrará en esta zona y por tanto no será ninguna de las inicialmente propuestas por el gobierno local, con lo que aparece por primera vez la capacidad de buscar soluciones nuevas, no previstas por la administración, por parte de los NIP. La aparente cara oscura de esta decisión fue que el emplazamiento elegido en primer lugar no pudo ser respetado, porque al realizar el correspondiente informe técnico se comprobó su escasa viabilidad. Sin embargo, una comisión de miembros del NIP fue informada del problema y ante los argumentos aceptó cambiar a la segunda propuesta más votada por el grupo. Aunque por problemas presupuestarios el campo no ha sido aún construido, ha desaparecido la incertidumbre sobre cual va a ser su emplazamiento definitivo, que hoy ya nadie discute.

\begin{tabular}{|l|c|c|c|}
\hline \multicolumn{4}{|c|}{ Caracteristicas descriptivas de tres NIPs } \\
\hline \multicolumn{1}{|c|}{ Población/Zona } & Idiazabal & Astigarraga & Guiprizcoa \\
\hline Tema & $\begin{array}{c}\text { Campo } \\
\text { futbol }\end{array}$ & $\begin{array}{c}\text { Instalación } \\
\text { Deportiva }\end{array}$ & Autovía \\
\hline $\begin{array}{l}\text { Institución } \\
\text { organizadora }\end{array}$ & Ayuntamiento & Ayuntamiento & Diputación \\
\hline $\begin{array}{l}\text { Número de NIPs } \\
\text { (participantes) }\end{array}$ & \begin{tabular}{c}
$(25)$ \\
\hline $\begin{array}{l}\text { Población } \\
\text { afectada }\end{array}$
\end{tabular} & $\begin{array}{c}2 \\
(50)\end{array}$ & $\begin{array}{c}14 \\
(303)\end{array}$ \\
\hline $\begin{array}{l}\text { Periodo de } \\
\text { realización }\end{array}$ & $7 / 92$ & $11 / 92$ & $\begin{array}{c}\text { Otoño 93/ } \\
\text { Primavera 94 }\end{array}$ \\
\hline Número de expertos & 5 & 5 & 13 \\
\hline $\begin{array}{l}\text { Número de sectores } \\
\text { opinantes. }\end{array}$ & 4 & 7 & 27 \\
\hline
\end{tabular}

La segunda experiencia tendrá lugar en un municipio ligeramente mayor, Astigarraga, una localidad de las afueras de Donostia, que ha ido desarrollando su propia personalidad y servicios a partir de la segregación de la capital. En este caso, el cambio de mayoría municipal es clave para entender la opción por el NIP. Un ayuntamiento de coalición EA-PNV sustituye a un gobierno local de $\mathrm{HB}$, que sigue siendo la primera fuerza electoral en el municipio. El nuevo equipo de gobierno quiere construir un frontón y unos nuevos equipamientos deportivos, pero en emplazamientos diferentes a los proyectados por el anterior ayuntamiento. Para prevenir conflictos y tratando de ampliar el acuerdo en torno al proyecto, se opta por un NIP para que tome la decisión.
En este caso el entorno va a ser más conflictivo y, aunque el dictamen final será aceptado por todos, habrá mayores reticencias al proceso por parte de algunos sectores. Como reflejo de este entorno más complejo, serán ya más numerosos y plurales los sectores que querrán participar en el proceso de opinar ante los miembros de los dos NIP que se constituyen en este caso. Quizás un factor importante para entender el éxito del proyecto se encuentre nuevamente en la capacidad de innovación de los NIP, que también en este caso propondrán una ubicación para el frontón que no coincide con ninguna de las propuestas por los técnicos municipales. El NIP decide que es preciso hacer el frontón y un polideportivo y propone una ubicación para cada uno de ellos. El primero está ya en funcionamiento y el segundo en construcción.

El tercer ejemplo será, con mucho, el más ambicioso y complejo. Iniciada su planificación en 1993 , no concluirá hasta casi un año después. Se tratará de programar las comunicaciones en el llamado eje Maltzaga-Urbina, que discurre desde la costa vasca hacia Vitoria, y cuyo trazado corresponde a la Diputación Foral de Guipúzcoa. Esta pretende construir una autovía en esta zona, aunque hay diversos trazados posibles, así como la opción por mejorar la carretera ya existente y abandonar el proyecto de autovía. Las implicaciones ecológicas y de patrimonio histórico que la decisión supone y el potencial de conflicto a que podian dar lugar, lleva a la Diputación a optar por realizar un NIP.

En este caso se tratará de 14 NIPs. La población a representar es mucho mayor, pues estamos hablando de 21 municipios potencialmente afectados, algunos tan importantes como Eibar, Vitoria o Donostia. Los NIPs se desarrollarám en 4 de estos municipios (pero con representación de todas las poblaciones) y a lo largo de 3 meses. La difusión del proyecto para que sea conocido en todas las localidades o las negociaciones para que el resultado sea aceptado por todos los actores serán tremendamente complejos. Procesos como la selección de los expertos o la distribución de palabras exigirán largas negociaciones, que demuestran la importancia de contar con un organizador independiente y con capacidad de diálogo. El conjunto de organizaciones que será escuchado por los miembros de los NIP será mucho más amplio que en los casos anteriores: cámaras de comercio, grupos ecologistas, ayuntamientos, particulares y organizaciones políticas, participarán en el proceso.

El dictamen final será también complejo. Existe un amplio consenso en la necesidad de mejorar las comunicaciones en la zona y una estrecha mayoría optará por la necesidad de construir una nueva autovía. Asimismo, los miembros optan por las características, las vías de financiación y los accesos que, a su juicio, y tras escuchar a todas las partes, debería tener dicha autovía. En este momento, los técnicos están terminando el trazado definitivo y el comentario general es que se encuentran 
con mucha menor oposición de la que sería esperable, si se tratara de una decisión tomada en solitario por la administración.

Además del resultado de los NIPs en términos de políticas concretas, es preciso señalar el impacto sobre sus propios participantes. El grado de satisfacción de los mismos con la experiencia era muy grande, siendo casi unánime en todos los casos. También en la gran mayoría de los casos, se consideraba que éste era un buen procedimiento para solucionar otros problemas y se consideraba que la ejecución había sido positiva y neutral (cuadro 2). Aunque no queda claro si el NIP ha tenido algún impacto a medio plazo en la conciencia ciudadana, al menos en el corto plazo, tanto los participantes como las instituciones mostraban una gran satisfacción con ese rol que se había asumido temporalmente: los ciudadanos satisfechos de haberse sentido "útiles e importantes", los políticos "comprendidos" cuando los ciudadanos habian experimentado el enfrentarse a la complejidad de los problemas y las decisiones colectivas.

\section{III. ¿Qué conclusiones podemos sacar sobre los NIPs a partir de estas experiencias?}

¿Son útiles los NIP? ¿Hay circunstancias idoneas para su utilización o condiciones que los convierten en inoperantes? ¿Vale la pena el esfuerzo dados los costes económicos y la complejidad de su organización? ¿En que circunstancias consiguen realmente ampliar la legitimidad de las decisiones adoptadas? Éstas son algunas de las preguntas que queremos plantearnos en este apartado, a partir del análisis de las condiciones concretas que acompañaron al desarrollo de las tres experiencias que acabamos de describir.

El Cuadro 2 sintetiza algunas de las características que distinguen a estos NIPs, en función de las condiciones políticas en que se desarrollan y de sus resultados. Sin entrar aún en detalles sobre cada uno de los indicadores, una lectura rápida del cuadro nos daría una primera conclusión: Idiazabal parece reunir las características del NIP ideal, mientras los dos otros casos, a pesar de su desarrollo básicamente positivo, presentan algunos pequeños inconvenientes, distintos en cada uno de los casos.

En Idiazabal el NIP se produce antes de que el tema se haya convertido en tema de debate público en la población y sin que el ayuntamiento tenga una postura definida sobre el tema. Esto lleva a una ausencia absoluta de conflicto en el proceso (favo- recida, sin duda, por la menor complejidad social de la población), a una clara mayoría en el seno del NIP, a una elevada legitimidad de la decisión y a una satisfacción extrema de los participantes en la experiencia. La muestra, además, va a sufrir menos los problemas que crean las sustituciones, dado que éstas han sido mucho menos necesarias que en los otros casos ${ }^{6}$.

\begin{tabular}{|l|c|c|c|}
\hline \multicolumn{3}{|c|}{ Cuadro 2 } \\
\hline \multicolumn{3}{|c|}{ Caracteristicas analiticas de tres NIPs } \\
\hline Visibilidad del problema & Preventivo & Emergente & Preventivo? \\
\hline Actitud de la Institución & Neutral & Decantada & Decantada \\
\hline $\begin{array}{l}\text { Decisión acorde con la Insti- } \\
\text { tución }\end{array}$ & - & Parcialmente & Sí \\
\hline $\begin{array}{l}\text { Tipo de mayoría en la deci- } \\
\text { sión }\end{array}$ & Clara & Ajustada & Ajustada \\
\hline $\begin{array}{l}\text { Aceptación social de la deci- } \\
\text { sión }\end{array}$ & Muy alta & Media/alta & Alta? \\
\hline $\begin{array}{l}\text { \% muy satisfechos con uso } \\
\text { del NIP }\end{array}$ & 84 & 74 & 46 \\
\hline $\begin{array}{l}\text { \% a favor otros usos de los } \\
\text { NIP }\end{array}$ & 100 & 94 & 75 \\
\hline $\begin{array}{l}\text { \% no satisfechos con los ex- } \\
\text { pertos }\end{array}$ & 1,1 & 2,1 & 5,6 \\
\hline $\begin{array}{l}\text { Índice de rechazo a partici- } \\
\text { par }\end{array}$ & 0 & 6 & 23 \\
\hline
\end{tabular}

En Astigarraga, a pesar de la valoración positiva ampliamente compartida, no todos los indicadores son coincidentes. Mientras el grado de satisfacción de los participantes es muy elevado en todos los aspectos, la unanimidad respecto al resultado de la decisión parece ser menor. Es preciso insistir aquí en que, dadas las condiciones de polarización potencial ante cualquier decisión política en el País Vasco y considerando que se está tomando una decisión en contra de la voluntad de la mayor de las minorías locales (HB), el NIP ha tenido el efecto importantísimo de evitar ninguna expresión de rechazo abierto a la decisión. Al mismo tiempo, sin embargo, se percibe en el ambiente que ésta ha sido una decisión donde ha habido vencedores y vencidos, y como consecuencia, mayorías más ajustadas y una actitud de triunfalismo de unos y de mayor escepticismo de otros ${ }^{8}$.

La situación es prácticamente la inversa en la autovía. Aunque es preciso ser prudente a la hora de hablar sobre el grado de aceptación de una medida que siempre puede resultar polémica y que no suele despertar mayores rechazos hasta que se inicia una construcción que hoy aún no ha empezado, la deci- 
sión parece contar con una amplia aceptación social, resultado en buena medida atribuible al procedimiento utilizado para tomarla. Por el contrario, los indicadores menos positivos aparecen aquí por parte de los participantes, cuya valoración de la experiencia sigue siendo positiva, aunque sin llegar a las unanimidades de los casos anteriores. Tanto la muchísima mayor complejidad del proceso y de los sectores participantes en él, como algunas de las polémicas que se produjeron a lo largo del mismo como resultado de esta misma diversidad, se encuentran sin duda en la raíz de estas valoraciones que, sin embargo, siguen siendo eminentemente positivas. La elevada proporción de sustituciones necesaria para completar la muestra en el area urbana nos muestra, una vez más, los problemas añadidos al movernos hacia escenarios de mayor complejidad social.

¿Podemos sacar alguna otra lección del conjunto de estas experiencias? Creo que, cuando menos, es necesario realizar alguna reflexión final en torno a dos grandes temas: las condiciones políticas ideales para la realización de un NIP, y los costes de estas operaciones y la valoración y las implicaciones que de éstos puede hacerse.

1) ¿Hay condiciones ideales?: Uno de los primeros debates que los NIP plantean es su posible ámbito de utilización. ¿Sirven para todos los problemas, para todo tipo de situaciones? Aunque el tema es demasiado nuevo para dar una respuesta categórica al respecto, cuestiones como las tratadas en las experiencias guipuzcoanas, que exigen respuestas limitadas y concretas son, probablemente, mucho más adecuadas que problemáticas más amplias y generales, como es el caso de un reciente NIP británico, que trataba de encontrar soluciones nada menos que a los problemas de la sanidad pública. Sin cerrar las puertas a experimentar su uso en cuestiones más generales y de respuesta más compleja, los NIP parecen más indicados para decidir la necesidad y el trazado de una autovía, que para proponer un nuevo marco de relaciones entre Escocia y Gran Bretaña.

Pero más que conclusiones sobre temas concretos, estas experiencias muestran las grandes diferencias entre NIPs, dependiendo de en qué estadio se encuentre el desarrollo del problema cuando éste es abordado por el grupo: el campo de futbol de Idiazabal no se había convertido aún en tema de debate público, los proyectos de autovía eran aún poco conocidos, aunque ya había posturas previas como resultado del debate generado sobre Leizarán y, en Astigarraga, sí había existido debate pues el anterior equipo municipal ya había manifestado su intención de renovar el frontón, abriéndose la discusión pública sobre el cómo y el dónde. La existencia de un debate, y de cierta polarización al respecto, llevó incluso a los organizadores del NIP a alterar una regla habitual en este procedimiento: pidieron a los participantes no comentar fuera del NIP lo que en éste se había discutido, hasta que el proceso hubiera terminado. De algún modo, se trataba de que esa "democracia deliberativa. que el NIP permite, esa reflexión y ese diálogo racional e informado que constituyen uno de sus pilares y de sus mejores argumentos, no fuera contaminado por el debate pasional y partidista de la calle, donde las identidades de cada uno con sus respectivos grupos sociales y líderes de opinión, podía primar por encima de esa voluntad de razonar y discutir libremente. Esta situación de posicionamientos previos lleva a que, a pesar del saldo globalmente positivo de la experiencia, ésta no alcance el mismo grado de unanimidad en sus resultados que en los otros casos.

En realidad, lo que se plantea es hasta qué punto ese diálogo y esa reflexión abiertos son fáciles, cuando los participantes llegan ya con ideas firmemente asentadas sobre cual es su posición ante el tema. ¿Puede la asistencia a un NIP llevar a cambiar de idea, a flexibilizar posiciones, a encontrar nuevas propuestas en estas circunstancias? En el caso de la autovía se formuló esta pregunta a los participantes tras la experiencia: dos de cada tres no tenían postura previa al llegar allí; del resto, dos de cada tres se habían mantenido en las mismas ideas que tenían al llegar y el resto las había modificado. No es un resultado que lleve a conclusiones pesimistas (o sea, bastante gente está dispuesta a cambiar de opinión tras nuevas informaciones y como resultado de la discusión), pero la posible autovía tampoco había sido un tema que hubiera generado un debate amplio y polarizado. Por poner un ejemplo claro, en un NIP formado por salmantinos y catalanes para tomar una decisión sobre los archivos de la guerra civil, ¿conseguirían los mejores y más neutrales historiadores y archiveros convencer a muchos de los primeros de que los documentos deberían ir a Cataluña o a muchos de los segundos de que éstos deberían permanecer en Salamanca?

En resumen, el posicionamiento previo de los actores políticos; la formación clara de un mapa de adversarios y defensores de determinada propuesta, dificulta en cierta medida el funcionamiento del NIP. Por ello, aunque en absoluto deba descartarse el uso de NIPs en torno a temas que ya han generado un amplio debate social, parece aconsejable su uso preventivo, antes de que un enquistamiento excesivo de las posturas cierre los caminos por los que debe circular el diálogo y la reflexión conjunta de los ciudadanos.

Estrechamente asociado al argumento anterior encontramos otro interrogante: ¿Debe la institución que promueve y paga el NIP ser neutral o puede a la vez ser uno de los actores implicados en el proceso y defender su propia opción? La respuesta no es fácil. Debería ser más fácil aceptar que la institución sea también una de las partes implicadas, dado que no interviene directamente en la operación, pues la presencia de un organizador profesional pretende precisamente garantizar la mayor neutralidad posible del proceso. Pero no podemos olvidar que, por mucha neutralidad y ética que le supongamos al organiza- 
dor, la institución es quien contrata y paga, y se hace difícil tratarla como a cualquier otro de los implicados. Por otro lado, negar que un NIP pueda servir por el hecho de que la institución tenga preferencias nos puede llevar a situaciones absurdas: a descartarlos en casos donde pueden ser de utilidad (Astigarraga o la autovía), o a la hipocresía de que las instituciones escondieran sus preferencias reales. Si una institución cree estar convencida de cual es la solución mejor, y aún así opta por utilizar un NIP para comprobar si esa decisión cuenta con el respaldo ciudadano, bienvenida sea la oportunidad participativa. A pesar de todo ello, no hay duda que la situación idílica es aquella donde el ayuntamiento no tiene preferencias (Idiazabal) porque así su actuación no despierta suspicacias, mientras que en los otros casos, éstas aparecen necesariamente a lo largo del proceso, aunque pueden ser corregidas con una actuación escrupulosamente neutral y suficientemente negociadora por parte de los responsables del NIP, tal como muy claramente se hizo en el caso de la autovía.

2) Un tema fundamental y que no puede obviarse en un análisis de los NIP es su coste económico. La necesidad de compensar a los participantes, el sueldo de los profesionales que lo organizan y los gastos de organización que implica, provocan un elevado coste total, que asusta y crea escepticismo a muchos de sus posibles usuarios. De hecho, la introducción del argumento económico al análisis de los casos aludidos nos llevaría quizás a modificar la percepción que hasta ahora habíamos construido: el retrato de Idiazabal como NIP ideal. Aunque no disponemos de los datos exactos sobre el coste, parece probable que el gasto generado por el NIP, tanto en relación a la población, como al coste de la implementación de la política que se está decidiendo, sea mayor en Idiazabal que en los casos restantes.

¿Por qué hablar tanto de los NIP entonces, si su precio los hace prohibitivos? Es lógico que el coste del NIP asuste al responsable de las finanzas en la institución que se plantea su uso. Más aún si tenemos en cuenta que la introducción de la voluntad popular llevará, en la mayor parte de los casos, a un proyecto de política más exigente y, por tanto, generalmente más caro.
Sin embargo, situaciones como las de Leizarán, donde los costes generados por la contestación que el proyecto despertó doblaron el precio de la obra, ilustran las ventajas económicas de prevenir el conflicto. No hay duda que, en ese caso, un NIP habría abaratado enormemente la construcción. Pero no es necesario recurrir a casos tan extremos y en cualquier presupuesto público podemos encontrar partidas añadidas para acallar potenciales conflictos. El coste del NIP puede ser una más de ellas, pues su eficacia en este terreno parece indudable.

Por otro lado, es obvio que precisamente por su costo, su complejidad y las implicaciones políticas que tiene, los NIP, al menos en su formulación actual, son un procedimiento útil como mecanismo extraordinario de toma de decisiones y no como un instrumento de participación regular de los ciudadanos en los procesos de toma de decisiones.

Pero creo que existe otro argumento clave contra las reticencias financieras que provocan los NIP. Los NIP no actúan sólo como mecanismos para tomar una decisión, sino como instrumentos de educación democrática. Los participantes reconocen abrumadoramente que su interés por los asuntos públicos se ha incrementado y los gobernantes se sienten más comprendidos cuando un conjunto de ciudadanos ha tenido que asumir responsabilidades que no les son propias y "mojarse" sobre qué terreno hay que expropiar si es preciso, sobre dónde hay que buscar los fondos para construir la autovía o sobre qué instalaciones no son imprescindibles en el polideportivo local. En definitiva, a través de los participantes y del efecto multiplicador que éstos puedan realizar al explicar su actividad, el NIP ha contribuido a que exista una mayor educación democrática, en el más amplio sentido de la palabra (participación, diálogo, responsabilidad). Y dada la crisis de credibilidad por la que pasan los representantes políticos en muchos de los países occidentales, no parece que mejorar esta relación entre ciudadanos y política sea un asunto secundario. En cualquier caso, ésta es quizás una de las grandes posibilidades de los NIP y por ello resulta particularmente importante que se profundice en investigar a lo que sus propios impulsores llaman asus efectos secundarios.
- Profesor de Ciencias Políticas. Universitat Autònoma de Barcelona.

1 Ver SuBirats (1996), en este mismo número, para los argumentos y la bibliografía.

2 Por supuesto, existen instrumentos tipo Consejo Económico y Social o Consejos locales de cultura, que cuentan con mayor formalización. Pero además de ser una muy pequeña parte del escenario, incluso en estos casos el debate se circunscribe a las minorias organizadas y los procesos finales de toma de decisiones no son inequívocos.
${ }^{3}$ El profesor Peter Dienel, de la Universidad de Wuppertal, ha sido su principal impulsor. Hans Harms ha sido el máximo responsable de su implementación en el País Vasco.

4 De hecho, buena parte de las observaciones que se realizan aqui proceden de un viaje organizado por la Diputación de Barcelona (a quien agradezco la invitación a participar en la visita) para conocer mejor dicho instrumento y evaluar su posible aplicación en su propio ámbito. 
'Población total de los territorios históricos de Guipúzcoa y Álava.

${ }^{6}$ Aunque los sustitutos provengan también de una muestra elegida al azar, siempre existe la duda de si aquellos que han rechazadado participar tendrán caracteristicas en común, que estén alterando la composición de la muestra. De hecho, éste fue el caso de Donostia, donde la gran cantidad de sustituciones dio lugar a una muestra con una gran sobrerepresentación de personas con estudios universitarios.

- Personas que por alguna razón declinan participar, sobre la muestra teórica de participantes. Es decir, en Idiazábal hubo 1,1 personas que rechazaron participar, por cada una que lo aceptó.

${ }^{8}$ Esto no es sólo visible en las mayorias más ajustadas que se producen en el NIP ( 26 votos sobre 50 a favor del emplazamiento elegido para el frontón, por 19 para la segunda opción), sino en la manera cómo el ayuntamiento habla del NIP como una victoria politica o en cómo la oposición más bien rehuye hablar del tema. 


\section{gestión y \\ política pública}

vol. V, núm. 1, México, primer semestre de 1996

\section{GESTIÓN Y POLÍTICA PÚBLICA}

IVES MENY Las políticas del mimetismo institucional.

NANCY BIRSDALL La desigualdad como limitación del crecimiento en América DAVID ROSS Latina.

Y RICHARD SABOT

\section{GESTIÓN Y ORGANIZACIÓN}

ANDREW M. PETTIGREW Investigación longitudinal en estudios de caso sobre el cambio organizacional.

\section{GESTIÓN REGIONAL Y LOCAL}

PETER WARD Problemas actuales en el gobierno y la administración de las megaciudades latinoamericanas.

\section{EXPERIENCIAS RELEVANTES}

PEDRO REYES ORTEGA Subsidios al ingreso o a la producción. ¿Dilemas de la política agrícola mexicana?

GERMAN ALARCO Límites económicos a la privatización portuaria: el caso TOSONI mexicano 1992-1995.

\section{POSICIONES E IDEAS}

BERNARDO KLIKSBERG Participación popular: algunos problemas estratégicos.

División de Administración Pública

Centro de Investigación y Docencia Económicas, A.C.

Carretera México-Toluca 3655 (km. 16.5)

Lomas de Santa Fe, Delegación Alvaro Obregón

Apartado Postal 10-883, 01210, México, D.F.

Tel. (5) 727-98-00, ext. 2228. Fax: (5) 727-98-73 